\title{
CLASSIFICATION OF FACTORABLE SURFACES IN THE PSEUDO-GALILEAN SPACE
}

\author{
Muhittin Evren Aydin, Alper Osman ÖĞRenmiş And Mahmut \\ ERGüT \\ Firat University and Namik Kemal University, Turkey
}

\begin{abstract}
In this paper, we introduce the factorable surfaces in the pseudo-Galilean space $\mathbb{G}_{3}^{1}$ and completely classify such surfaces with null Gaussian and mean curvature. Also, in a special case, we investigate the factorable surfaces which fulfill the condition that the ratio of the Gaussian curvature and the mean curvature is constant in $\mathbb{G}_{3}^{1}$.
\end{abstract}

\section{INTRODUCTION}

The geometry of Galilean Relativity acts like a "bridge" from Euclidean geometry to Special Relativity. The Galilean space which can be defined in three-dimensional projective space $P_{3}(\mathbb{R})$ is the space of Galilean Relativity. The geometries of Galilean space and pseudo-Galilean space have similarities, but, of course, are different. In the Galilean and the pseudo Galilean space, surfaces of revolution, ruled surfaces, translation surfaces and tubular surfaces have been studied in $[3-5,10,11,13-15,17,20]$. For further study of surfaces in the pseudo Galilean space, we refer the reader to Šipuš and Divjak's paper [15].

One of the main problems in classical differential geometry of surfaces is to classify ones with null curvature. In particular, a surface is said to be developable if it has null Gaussian curvature (NGC). In this case the surface can be flattened onto a plane without distortion. We remark that cylinders and cones are examples of developable surfaces.

There exist remarkable applications of the results obtained on the surfaces of null curvature in different fields, for example in microeconomics. When

2010 Mathematics Subject Classification. 53A35, 53B30.

Key words and phrases. Factorable surface, Gaussian curvature, mean curvature, minimal surface, pseudo-Euclidean plane, pseudo-Galilean space. 
the graphs of production functions in microeconomics have NGC, one can realize a "good" analysis of isoquants by projections, without losing essential information about their geometry (see $[1,19])$. By motivating these, we target to study the factorable surfaces in the pseudo Galilean space $\mathbb{G}_{3}^{1}$.

In this paper, we classify the factorable surfaces with NGC and minimal ones in $\mathbb{G}_{3}^{1}$. Then, in a special case, we obtain a non-existence result for the factorable surfaces satisfying the condition that the ratio of the Gaussian curvature and the mean curvature is constant in $\mathbb{G}_{3}^{1}$.

\section{Preliminaries}

The pseudo-Galilean space $\mathbb{G}_{3}^{1}$ is one of the Cayley-Klein spaces with absolute figure that consists of the ordered triple $\{\omega, f, I\}$, where $\omega$ is the absolute plane in the three dimensional real projective space $P_{3}(\mathbb{R}), f$ the absolute line in $\omega$ and $I$ the fixed hyperbolic involution of points of $f$.

Homogenous coordinates of $\mathbb{G}_{3}^{1}$ can be given in the following way: The absolute plane $\omega$ is given by $x_{0}=0$, the absolute line $f$ by $x_{0}=x_{1}=0$ and the hyperbolic involution by $\left(0: 0: x_{2}: x_{3}\right) \longmapsto\left(0: 0: x_{3}: x_{2}\right)$, which is equivalent to the requirement that the conic $x_{2}^{2}-x_{3}^{2}=0$ is the absolute conic. The metric connections in $\mathbb{G}_{3}^{1}$ are introduced with respect to the absolute figure. In terms of the affine coordinates given by $\left(x_{0}: x_{1}: x_{2}: x_{3}\right)=(1: x: y: z)$, the distance between the points $X=\left(x_{1}, x_{2}, x_{3}\right)$ and $Y=\left(y_{1}, y_{2}, y_{3}\right)$ is defined by (see $[15,20])$

$$
d(X, Y)= \begin{cases}\left|y_{1}-x_{1}\right|, & \text { if } x_{1} \neq y_{1}, \\ \sqrt{\left|\left(y_{2}-x_{2}\right)^{2}-\left(y_{3}-x_{3}\right)^{2}\right|}, & \text { if } x_{1}=y_{1} .\end{cases}
$$

The pseudo-Galilean scalar product of the vectors $X=\left(x_{1}, x_{2}, x_{3}\right)$ and $Y=$ $\left(y_{1}, y_{2}, y_{3}\right)$ is defined by

$$
X \cdot Y= \begin{cases}x_{1} y_{1}, & \text { if } x_{1} \neq 0 \text { or } y_{1} \neq 0, \\ x_{2} y_{2}-x_{3} y_{3}, & \text { if } x_{1}=0 \text { and } y_{1}=0 .\end{cases}
$$

In this sense, the pseudo-Galilean norm of a vector $X$ is $\|X\|=\sqrt{|X \cdot X|}$. A vector $X=\left(x_{1}, x_{2}, x_{3}\right)$ is called isotropic (non-isotropic) if $x_{1}=0\left(x_{1} \neq 0\right)$. All unit non-isotropic vectors are of the form $\left(1, x_{2}, x_{3}\right)$. The isotropic vector $X=\left(0, x_{2}, x_{3}\right)$ is called spacelike, timelike and lightlike if $x_{2}^{2}-x_{3}^{2}>0, x_{2}^{2}-x_{3}^{2}<$ 0 and $x_{2}= \pm x_{3}$, respectively. The pseudo-Galilean cross product of $X$ and $Y$ on $\mathbb{G}_{3}^{1}$ is given by

$$
X \times Y=\left|\begin{array}{ccc}
0 & -e_{2} & e_{3} \\
x_{1} & x_{2} & x_{3} \\
y_{1} & y_{2} & y_{3}
\end{array}\right|
$$

where $e_{i}=\left(\delta_{i 1}, \delta_{i 2}, \delta_{i 3}\right), i=2,3$. 
Let $M^{2}$ be a surface in the pseudo-Galilean space $\mathbb{G}_{3}^{1}$ parametrized by

$$
r\left(u_{1}, u_{2}\right)=\left(r_{1}\left(u_{1}, u_{2}\right), r_{2}\left(u_{1}, u_{2}\right), r_{3}\left(u_{1}, u_{2}\right)\right) .
$$

Denote $\left(r_{i}\right)_{u_{j}}=\partial r_{i} / \partial u_{j}$ and $\left(r_{i}\right)_{u_{j} u_{k}}=\partial^{2} r_{i} / \partial u_{j} \partial u_{k}, i=1,2,3$ and $j, k=$ 1,2 . Then such a surface is admissible if and only if $\left(r_{1}\right)_{u_{j}}=\partial r_{1} / \partial u_{j} \neq 0$ for some $j=1,2$. The coefficients of the first fundamental form of $M^{2}$ are

$$
g_{i}=\left(r_{1}\right)_{u_{i}} \text { and } h_{i j}=\left(0,\left(r_{2}\right)_{u_{i}},\left(r_{3}\right)_{u_{i}}\right) \cdot\left(0,\left(r_{2}\right)_{u_{j}},\left(r_{3}\right)_{u_{j}}\right), i, j=1,2 \text {, }
$$

and, in matrix form, it can be written as

$$
d s^{2}=\left(\begin{array}{cc}
d s_{1}^{2} & 0 \\
0 & d s_{2}^{2}
\end{array}\right),
$$

where $d s_{1}^{2}=\left(g_{1} d u_{1}+g_{2} d u_{2}\right)^{2}$ and $d s_{2}^{2}=h_{11} d u_{1}^{2}+2 h_{12} d u_{1} d u_{2}+h_{22} d u_{2}^{2}$.

Let us define the function $W$ as

$$
W=\sqrt{\left|\left(\left(r_{1}\right)_{u_{1}}\left(r_{2}\right)_{u_{2}}-\left(r_{1}\right)_{u_{2}}\left(r_{2}\right)_{u_{1}}\right)^{2}-\left(\left(r_{1}\right)_{u_{1}}\left(r_{3}\right)_{u_{2}}-\left(r_{1}\right)_{u_{2}}\left(r_{3}\right)_{u_{1}}\right)^{2}\right|} .
$$

Then the normal vector field $N$ of $M^{2}$ is given by

$$
N=\frac{1}{W}\left(0,\left(r_{1}\right)_{u_{1}}\left(r_{3}\right)_{u_{2}}-\left(r_{1}\right)_{u_{2}}\left(r_{3}\right)_{u_{1}},\left(r_{1}\right)_{u_{1}}\left(r_{2}\right)_{u_{2}}-\left(r_{1}\right)_{u_{2}}\left(r_{2}\right)_{u_{1}}\right),
$$

where $N \cdot N=\varepsilon= \pm 1$. Hence, two types of admissible surfaces can be distinguished: spacelike surfaces having timelike unit normals $(\epsilon=-1)$ and timelike ones having spacelike unit normals $(\epsilon=1)$.

The coefficients of the second fundamental form of $M^{2}$ are

$$
\begin{aligned}
L_{i j} & =\varepsilon \frac{1}{g_{1}}\left(g_{1}\left(0,\left(r_{2}\right)_{u_{i} u_{j}},\left(r_{3}\right)_{u_{i} u_{j}}\right)-\left(g_{i}\right)_{u_{j}}\left(0,\left(r_{2}\right)_{u_{1}},\left(r_{3}\right)_{u_{1}}\right)\right) \cdot N \\
& =\varepsilon \frac{1}{g_{2}}\left(g_{2}\left(0,\left(r_{2}\right)_{u_{i} u_{j}},\left(r_{3}\right)_{u_{i} u_{j}}\right)-\left(g_{i}\right)_{u_{j}}\left(0,\left(r_{2}\right)_{u_{2}},\left(r_{3}\right)_{u_{2}}\right)\right) \cdot N .
\end{aligned}
$$

The Gaussian curvature and the mean curvature of $M^{2}$ are respectively defined by

$$
K=-\varepsilon \frac{L_{11} L_{22}-L_{12}^{2}}{W^{2}}
$$

and

$$
H=-\varepsilon \frac{g_{2}^{2} L_{11}-2 g_{1} g_{2} L_{12}+g_{1}^{2} L_{22}}{2 W^{2}} .
$$

A surface in $\mathbb{G}_{3}^{1}$ is said to be minimal if its mean curvature vanishes. 


\section{Factorable surfaces With NGC in $\mathbb{G}_{3}^{1}$}

A surface $M^{2}$ in the pseudo-Galilean space $\mathbb{G}_{3}^{1}$ is called a factorable surface if it can be locally written as

$$
r\left(u_{1}, u_{2}\right):=\left(u_{1}, u_{2}, f_{1}\left(u_{1}\right) f_{2}\left(u_{2}\right)\right),
$$

or

$$
r\left(u_{1}, u_{3}\right):=\left(u_{1}, f_{1}\left(u_{1}\right) f_{3}\left(u_{3}\right), u_{3}\right),
$$

or

$$
r\left(u_{2}, u_{3}\right):=\left(f_{2}\left(u_{2}\right) f_{3}\left(u_{3}\right), u_{2}, u_{3}\right),
$$

where $f_{i}$ are smooth functions of one variable, for all $i \in\{1,2,3\}$. We call the surfaces given by (3.1), (3.2) and (3.3) the factorable surfaces of the first type, the second type and the third type, respectively. The factorable surfaces in the Euclidean space, the pseudo-Euclidean space and the Heisenberg group have been studied in $[2,12,18,21]$.

The following result provides a complete classification of the factorable surfaces having NGC in $\mathbb{G}_{3}^{1}$.

THEOREM 3.1. Let $M^{2}$ be a factorable surface with $N G C$ in $\mathbb{G}_{3}^{1}$. If $M^{2}$ is a factorable surface of the first type (respectively the second type and the third type), then either

(a) at least one of $f_{1}, f_{2}$ (respectively $f_{1}, f_{3}$ and $f_{2}, f_{3}$ ) is a constant function, or

(b) $f_{i}\left(u_{i}\right)=\gamma_{i} e^{\beta_{i} u_{i}}$, where $\beta_{i}, \gamma_{i} \in \mathbb{R} \backslash\{0\}, i \in\{1,2\}$, (respectively $i \in$ $\{1,3\}$ and $i \in\{2,3\})$ or

(c) $f_{i}\left(u_{i}\right)=\left[\left(1-\alpha_{i}\right) \zeta_{i} u_{i}+\lambda_{i}\right]^{\frac{1}{1-\alpha_{i}}}$, where $\alpha_{i} \neq 0,1, \alpha_{i} \in \mathbb{R}$ and $\alpha_{i} \alpha_{j}=$ $1, \zeta_{i} \in \mathbb{R} \backslash\{0\}$ and $\lambda_{i} \in \mathbb{R}, i \in\{1,2\}$ (respectively $i \in\{1,3\}$ and $i \in\{2,3\})$.

Conversely, the factorable surfaces satisfying the conditions in cases (a)-(c) have NGC.

Proof. It is only proved for the factorable surfaces of the first type since one can be verified by using the following way for the factorable surfaces of the second and the third types.

Let $M^{2}$ be a factorable surface of the first type in $\mathbb{G}_{3}^{1}$. Then the coefficients of the first and the second fundamental forms of $M^{2}$ are

$$
\begin{aligned}
& g_{1}=1, \quad g_{2}=0, \\
& h_{11}=-\left(f_{1}^{\prime} f_{2}\right)^{2}, \quad h_{12}=-\left(f_{1} f_{2}\right) f_{1}^{\prime} f_{2}^{\prime}, \quad h_{22}=1-\left(f_{1} f_{2}^{\prime}\right)^{2}, \\
& N=\frac{\left(0, f_{1} f_{2}^{\prime}, 1\right)}{W}, \\
& L_{11}=\frac{-\varepsilon}{W}\left(f_{1}^{\prime \prime} f_{2}\right), \quad L_{12}=\frac{-\varepsilon}{W}\left(f_{1}^{\prime} f_{2}^{\prime}\right), \quad L_{22}=\frac{-\varepsilon}{W}\left(f_{1} f_{2}^{\prime \prime}\right),
\end{aligned}
$$


where $f_{i}^{\prime}=\frac{d f_{i}}{d u_{i}}, f_{i}^{\prime \prime}=\frac{d^{2} f_{i}}{d u_{i}^{2}}, i \in\{1,2\}$, and $W=\sqrt{\left|1-\left(f_{1} f_{2}^{\prime}\right)^{2}\right|}$. It can be easily seen that the Gaussian curvature of $M^{2}$ is

$$
K=\frac{-\varepsilon}{W^{4}}\left[\left(f_{1}^{\prime \prime} f_{2}^{\prime \prime}\right) f_{1} f_{2}-\left(f_{1}^{\prime} f_{2}^{\prime}\right)^{2}\right] .
$$

Now, let us assume that $M^{2}$ has NGC. It immediately implies that $f_{1}$ or $f_{2}$ is a constant function which gives case (a). If $f_{1}$ and $f_{2}$ are non-constant functions, then by (3.5), we get

$$
\left(f_{1}^{\prime \prime} f_{2}^{\prime \prime}\right) f_{1} f_{2}-\left(f_{1}^{\prime} f_{2}^{\prime}\right)^{2}=0 .
$$

Since $f_{i}^{\prime \prime}, i \in\{1,2\}$, cannot be zero, the equation (3.6) can be written as

$$
\frac{f_{1}^{\prime \prime} f_{1}}{\left(f_{1}^{\prime}\right)^{2}}-\frac{\left(f_{2}^{\prime}\right)^{2}}{f_{2}^{\prime \prime} f_{2}}=0 .
$$

Thus equation (3.7) yields the following ordinary differential equation (ODE)

$$
\frac{f_{i}^{\prime \prime} f_{i}}{\left(f_{i}^{\prime}\right)^{2}}=\alpha_{i}, \quad i \in\{1,2\}
$$

for $\alpha_{i} \in \mathbb{R} \backslash\{0\}$ and $\alpha_{i} \alpha_{j}=1$. Equation (3.8) can be reduced to the following Bernoulli ODE

$$
f_{i}^{\prime}-\beta_{i} f_{i}^{\alpha_{i}}=0, \beta_{i} \in \mathbb{R} \backslash\{0\}, i \in\{1,2\} .
$$

For $\alpha_{i}=1, i \in\{1,2\}$, after solving the ODE (3.9), we get

$$
f_{i}\left(u_{i}\right)=\gamma_{i} e^{\beta_{i} u_{i}}, \gamma_{i} \in \mathbb{R} \backslash\{0\}, i \in\{1,2\},
$$

which gives case (b). If $\alpha_{i} \neq 1, i \in\{1,2\}$, then by solving the ODE (3.9), we obtain

$$
f_{i}\left(u_{i}\right)=\left[\left(1-\alpha_{i}\right) \zeta_{i} u_{i}+\lambda_{i}\right]^{\frac{1}{1-\alpha_{i}}},
$$

where $\alpha_{i} \in \mathbb{R} \backslash\{0\}$ and $\alpha_{i} \alpha_{j}=1, \zeta_{i} \in \mathbb{R} \backslash\{0\}$ and $\lambda_{i} \in \mathbb{R}$, for $i \in\{1,2\}$, which proves case (c).

Conversely, it is direct to verify that all of the factorable surfaces given by in cases (a)-(c) have NGC.

EXAMPLE 3.2. Let $M^{2}$ be a factorable surface of the first type having NGC in $\mathbb{G}_{3}^{1}$. Taking the parametrizations $u_{3}=0.5\left(u_{1}\right)^{0.5}, u_{3}=$ $1.5 \exp \left(u_{1}+u_{2}\right)-5$ and $u_{3}=-0.25\left(u_{1}\right)^{-1}\left(u_{2}\right)^{2}$ defined as in three cases of Theorem 3.1, then we respectively draw those as in Figure 1, Figure 2, Figure 3. 


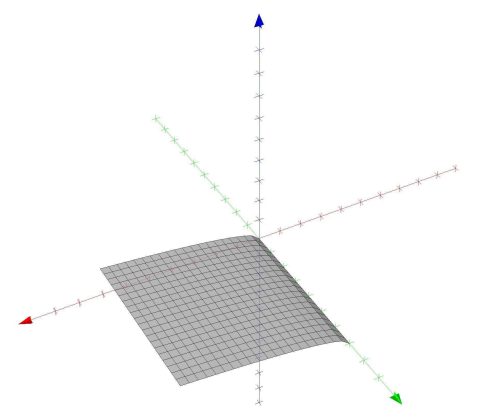

FiguRE 1. The factorable surface having NGC with $u_{3}=0.5\left(u_{1}\right)^{0.5}$.

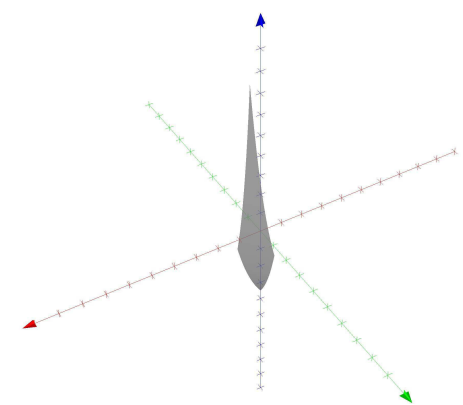

Figure 2. The factorable surface having NGC with $u_{3}=$ $1.5 \exp \left(u_{1}+u_{2}\right)-5$.

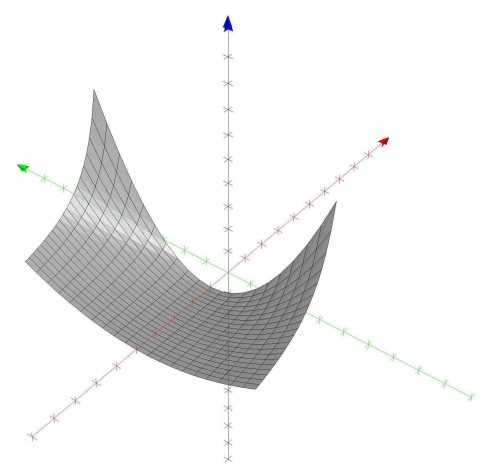

FIGURE 3. The factorable surface having NGC with $u_{3}=-0.25\left(u_{1}\right)^{-1}\left(u_{2}\right)^{2}$.

4. Minimal factorable surfaces in $\mathbb{G}_{3}^{1}$

Next theorem provides a classification for minimal factorable surfaces in $\mathbb{G}_{3}^{1}$. 
THEOREM 4.1. Let $M^{2}$ be a factorable surface in $\mathbb{G}_{3}^{1}$. Then $M^{2}$ is minimal if and only if one of the following statements holds:

(a) when $M^{2}$ is of the first type, $f_{2}$ is a linear function;

(b) when $M^{2}$ is of the second type, $f_{3}$ is a linear function;

(c) when $M^{2}$ is of the third type, either

(c.i) at least one of $f_{2}$ and $f_{3}$ is a constant function, or

(c.ii) $f_{i}\left(u_{i}\right)=\gamma_{i} e^{\beta_{i} u_{i}}$, where $\beta_{i}, \gamma_{i} \in \mathbb{R} \backslash\{0\}, i \in\{2,3\}$, or

(c.iii) $f_{i}\left(u_{i}\right)=\left[\left(1-\alpha_{i}\right) \zeta_{i} u_{i}+\lambda_{i}\right]^{\frac{1}{1-\alpha_{i}}}$, where $\alpha_{i} \neq 1, \alpha_{i} \in \mathbb{R}$ and $\alpha_{2}+\alpha_{3}=2, \zeta_{i} \in \mathbb{R} \backslash\{0\}$ and $\lambda_{i} \in \mathbb{R}, i \in\{2,3\}$.

Proof. Let $M^{2}$ be a factorable surface of the first type, i.e. it is parametrized by (3.1). It follows from (3.4) that the mean curvature of $M^{2}$ is

$$
H=\frac{1}{2 W^{3}}\left[f_{1} f_{2}^{\prime \prime}\right] .
$$

Equation (4.1) implies that $M^{2}$ is minimal if and only if $f_{2}$ is a linear function. Similarly, it is not hard to prove that $f_{3}$ is a linear function when the factorable surface of the second type is minimal. This proves case (a) and case (b).

Now, let $M^{2}$ be a factorable surface of the third type. Then the coefficients of first and second fundamental forms of $M^{2}$ are

$$
\begin{aligned}
& g_{1}=f_{2}^{\prime} f_{3}, \quad g_{2}=f_{2} f_{3}^{\prime}, \quad h_{11}=1, \quad h_{12}=0, \quad h_{22}=-1, \\
& N=\frac{\left(0, f_{2}^{\prime} f_{3},-f_{2} f_{3}^{\prime}\right)}{W}, \\
& L_{11}=\frac{-\varepsilon}{W}\left(f_{2}^{\prime \prime} f_{3}\right), \quad L_{12}=\frac{-\varepsilon}{W}\left(f_{2}^{\prime} f_{3}^{\prime}\right), \quad L_{22}=\frac{-\varepsilon}{W}\left(f_{2} f_{3}^{\prime \prime}\right),
\end{aligned}
$$

where $W=\sqrt{\left|\left(f_{2}^{\prime} f_{3}\right)^{2}-\left(f_{2} f_{3}^{\prime}\right)^{2}\right|}$. From (4.2), the mean curvature of $M^{2}$ is

$$
H=\frac{1}{2 W^{3}}\left[\left(f_{2} f_{3}^{\prime}\right)^{2} f_{2}^{\prime \prime} f_{3}-2\left(f_{2}^{\prime} f_{3}^{\prime}\right)^{2}\left(f_{2} f_{3}\right)+\left(f_{2}^{\prime} f_{3}\right)^{2} f_{2} f_{3}^{\prime \prime}\right] .
$$

If $M^{2}$ is minimal, then (4.3) implies that

$$
\left(f_{2} f_{3}^{\prime}\right)^{2} f_{2}^{\prime \prime} f_{3}-2\left(f_{2}^{\prime} f_{3}^{\prime}\right)^{2}\left(f_{2} f_{3}\right)+\left(f_{2}^{\prime} f_{3}\right)^{2} f_{2} f_{3}^{\prime \prime}=0 .
$$

If one of the $f_{2}, f_{3}$ is a constant function, then the previous formula holds. This gives the proof of case (c.i). Otherwise, we conclude

$$
\frac{f_{2}^{\prime \prime} f_{2}}{\left(f_{2}^{\prime}\right)^{2}}+\frac{f_{3}^{\prime \prime} f_{3}}{\left(f_{3}^{\prime}\right)^{2}}=2
$$

We divide the rest part of proof into two cases:

CASE (1): $f_{i}^{\prime \prime} f_{i} /\left(f_{i}^{\prime}\right)^{2}=1$ for $i \in\{2,3\}$. Then it is easily seen that $f_{i}\left(u_{i}\right)=\gamma_{i} e^{\beta_{i} u_{i}}$ for $\beta_{i}, \gamma_{i} \in \mathbb{R} \backslash\{0\}, i \in\{2,3\}$, which gives case (c.ii). 
CASE (2): $f_{i}^{\prime \prime} f_{i} /\left(f_{i}^{\prime}\right)^{2} \neq 1$ for $i \in\{2,3\}$. From (4.4), we get

$$
\frac{f_{i}^{\prime \prime} f_{i}}{\left(f_{i}^{\prime}\right)^{2}}=\alpha_{i}, \alpha_{i} \in \mathbb{R}, \alpha_{i} \neq 1, i \in\{2,3\},
$$

such that $\alpha_{2}+\alpha_{3}=2$. After solving the ODE (4.5), we derive

$$
f_{i}\left(u_{i}\right)=\left[\left(1-\alpha_{i}\right) \zeta_{i} u_{i}+\lambda_{i}\right]^{\frac{1}{1-\alpha_{i}}},
$$

where $\zeta_{i} \in \mathbb{R} \backslash\{0\}$ and $\lambda_{i} \in \mathbb{R}, i \in\{2,3\}$. This completes to prove the case (c.iii).

Conversely, it is easy to verify that all of the factorable surfaces given by in cases (a)-(c.iii) are minimal.

ExAmple 4.2. Let $M^{2}$ be a minimal factorable surface in $\mathbb{G}_{3}^{1}$. Choosing $u_{3}=\cos \left(u_{1}\right)\left(u_{2}+1\right), u_{2}=\sin \left(u_{1}\right)\left(u_{3}+1\right)$ and $u_{1}=\left(2 u_{2}\right)^{0.5}\left(-2 u_{3}\right)^{-0.5}$ given as in case (a), case (b) and case (c.iii) of Theorem 4.1, we respectively draw ones as in Figure 4, Figure 5, Figure 6.

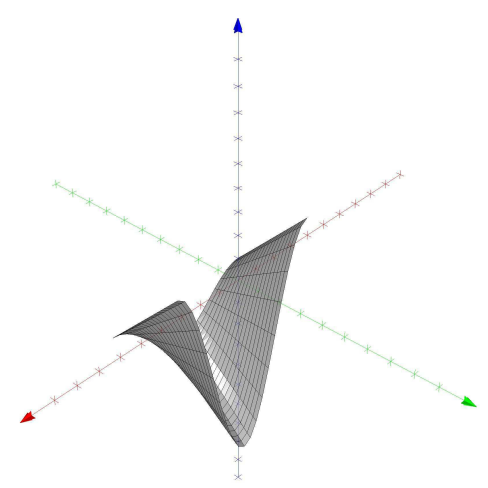

Figure 4 . The minimal factorable surface of the first type with $u_{3}=\cos \left(u_{1}\right)\left(u_{2}+1\right)$.

On the other hand, the authors in [8] introduced a new kind of curvature for the hypersurfaces of Euclidean $(n+1)$-spaces, called by amalgamatic curvature and explored its geometric meaning by proving an inequality related to the absolute mean curvature of the hypersurface. In three dimensional case, the amalgamatic curvature is indeed the harmonic ratio of the principal curvatures of any given surface, i.e., the ratio of the Gaussian curvature and the mean curvature.

Therefore, in the special case that $f_{1}$ is a linear function, we are able to analyze the factorable surfaces of first and second type having the ratio $K / H=$ const. $\neq 0$. For this purpose, let $M^{2}$ be a factorable surfaces of the first type. Take $f_{1}\left(u_{1}\right)=\alpha_{1} u_{1}+\beta_{1}$. Because of the Theorem 4.1, $f_{2}$ cannot 


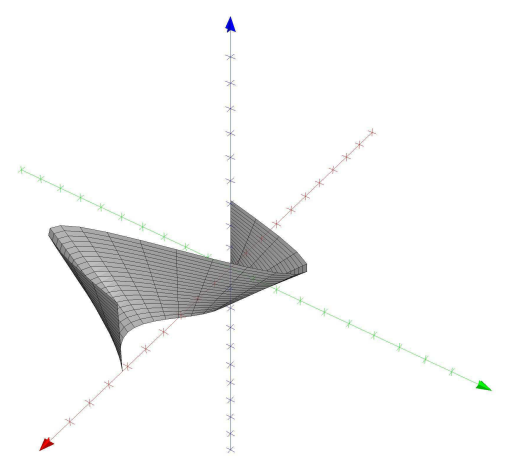

FiguRE 5. The minimal factorable surface of the second type with $u_{2}=\sin \left(u_{1}\right)\left(u_{3}+1\right)$.

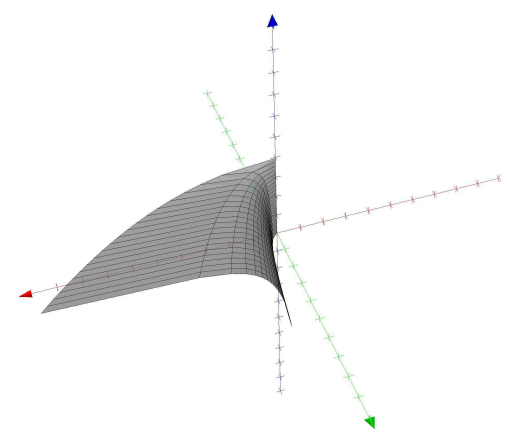

FiguRE 6 . The minimal factorable surface of the third type with $u_{1}=\left(2 u_{2}\right)^{0.5}\left(-2 u_{3}\right)^{-0.5}$.

be linear function. Then from (3.5) and (4.1), the ratio of the Gaussian curvature and the mean curvature of $M^{2}$ is

$$
\frac{K}{H}=\frac{1}{2 W} \frac{\alpha_{1}^{2}\left(f_{2}^{\prime}\right)^{2}}{\left(\alpha_{1} u_{1}+\beta_{1}\right) f_{2}^{\prime \prime}} .
$$

Suppose that $\frac{K}{H}=\lambda=$ const. Then we get

$$
\frac{1}{2 W} \frac{\alpha_{1}^{2}\left(f_{2}^{\prime}\right)^{2}}{\left(\alpha_{1} u_{1}+\beta_{1}\right) f_{2}^{\prime \prime}}=\lambda
$$

Taking the partial derivative of (4.6) with respect to $u_{1}$, we derive

$$
\left|1-\left(f_{1} f_{2}^{\prime}\right)^{2}\right|-\left(f_{1} f_{2}^{\prime}\right)^{2}=0 .
$$


If $M^{2}$ is timelike, then $\left(f_{1} f_{2}^{\prime}\right)^{2}-1<0$, which yields a contradiction. Otherwise, (4.7) implies that

$$
\left(\alpha_{1} u_{1}+\beta_{1}\right)^{2}\left(f_{2}^{\prime}\right)^{2}=\frac{1}{2} .
$$

Again by taking partial derivative of (4.8) with respect to $u_{1}$, we obtain

$$
\alpha_{1}\left(\alpha_{1} u_{1}+\beta_{1}\right)\left(f_{2}^{\prime}\right)^{2}=0,
$$

which is not possible. This implies that there does not exist a factorable surface of the first type fulfilling the ratio $K / H=$ const. $\neq 0$ in $\mathbb{G}_{3}^{1}$. Similarly, one can also be shown for the factorable surfaces of the second type.

Therefore, we have proved the following.

Corollary 4.3. Let $f_{1}$ be a linear function. Then there does not exist a factorable surface of the first and the second type fulfilling the ratio $\mathrm{K} / \mathrm{H}=$ const. $\neq 0$ in $\mathbb{G}_{3}^{1}$.

\section{ACKNOWLEDGEMENTS.}

The authors would like to thank the referee for valuable comments and suggestions which helped to improve the paper. All figures in this article are made with VisuMath (www.visumath.be).

\section{REFERENCES}

[1] M. E. Aydin and A. Mihai, Classifications of quasi-sum production functions with Allen determinants, Filomat 29(6) (2015), 1351-1359.

[2] M. Bekkar and B. Senoussi, Factorable surfaces in the three-dimensional Euclidean and Lorentzian spaces satisfying $\triangle r_{i}=\lambda_{i} r_{i}$, J. Geom. 103 (2012), 17-29.

[3] B. Divjak and Ž. M. Šipuš, Some special surfaces in the pseudo-Galilean space, Acta Math. Hungar. 118 (2008), 209-226.

[4] B. Divjak and Ž. M. Šipuš, Minding isometries of ruled surfaces in pseudo-Galilean space, J. Geom. 77 (2003), 35-47.

[5] B. Divjak and Ž. M. Šipuš, Special curves on ruled surfaces in Galilean and pseudoGalilean spaces, Acta Math. Hungar. 98 (2003), 203-215.

[6] B.-Y. Chen, Geometry of submanifolds, M. Dekker, New York, 1973.

[7] M. P. do Carmo, Differential geometry of curves and surfaces, Prentice Hall, Englewood Cliffs, NJ, 1976.

[8] C. T. R. Conley, R. Etnyre, B. Gardener, L. H. Odom and B. D. Suceavă, New curvature inequalities for hypersurfaces in the Euclidean ambient space, Taiwanese J. Math. 17(3) (2013), 885-895.

[9] M. Dede, Tubular surfaces in Galilean space, Math. Commun. 18 (2013), 209-217.

[10] I. Kamenarović, Existence theorems for ruled surfaces in the Galilean space $\mathbb{G}_{3}$, Rad Hrvatske Akad. Znan. Umjet. No. 456 (1991), 183-196.

[11] M. K. Karacan and Y. Tuncer, Tubular surfaces of Weingarten types in Galilean and pseudo-Galilean, Bull. Math. Anal. Appl. 5 (2013), 87-100.

[12] H. Meng and H. Liu, Factorable surfaces in 3-Minkowski space, Bull. Korean Math. Soc. 46 (2009), 155-169.

[13] Ž. Milin Šipuš, Ruled Weingarten surfaces in the Galilean space, Period. Math. Hungar. 56 (2008), 213-225. 
[14] Ž. Milin Šipuš and B. Divjak, Translation surface in the Galilean space, Glas. Mat. Ser. III 46(66) (2011), 455-469.

[15] Ž. Milin Šipuš and B. Divjak, Surfaces of constant curvature in the pseudo-Galilean space, Int. J. Math. Math. Sci. 2012, Art ID375264, 28pp.

[16] A. O. Öğrenmiş, M. Ergüt and M. Bektaş, On the helices in the Galilean space $\mathbb{G}_{3}$, Iran. J. Sci. Technol. Trans. A Sci. 31 (2007), 177-181.

[17] A. O. Öğrenmiş and M. Ergüt, On the Gauss map of ruled surfaces of type II in 3-dimensional pseudo-Galilean space, Bol. Soc. Parana. Mat. (3) 31 (2013), 145-152.

[18] E. Turhan, G. Altay, Maximal and minimal surfaces of factorable surfaces in Heis 3 , Int. J. Open Probl. Comput. Sci. Math. 3 (2010), 200-212.

[19] G. E. Vilcu, A geometric perspective on the generalized Cobb-Douglas production functions, Appl. Math. Lett. 24 (2011), 777-783.

[20] D. W. Yoon, Surfaces of revolution in the three dimensional pseudo-Galilean space, Glas. Mat. Ser. III 48(68) (2013), 415-428.

[21] Y. Yu and H. Liu, The factorable minimal surfaces, in Proceedings of the Eleventh International Workshop on Differential Geometry, Kyungpook Nat. Univ., Taegu, 2007, 33-39.

M. E. Aydin

Department of Mathematics

Firat University

23200 Elazig

Turkey

E-mail: meaydin@firat.edu.tr

A. O. Öğrenmiş

Department of Mathematics

Firat University

23200 Elazig

Turkey

E-mail: aogrenmis@firat.edu.tr

M. Ergüt

Department of Mathematics

Namik Kemal University

59000 Tekirdag

Turkey

E-mail: mergut@nku.edu.tr

Received: 15.10.2014.

Revised: 1.6.2015. 